\title{
Effect of Local Injection of 1, 25 Dihydroxycholecalciferol on Bone Remodeling in Uremic Rabbit Model
}

Marwa K. Khairallah* 1, Nessren M. Abd El-Rady², Yasmine S. Makarem ${ }^{3}$, Marwa A. Dahpy ${ }^{4}$, Hamdy M. Ibrahim ${ }^{5}$, Mostafa K. Abdo ${ }^{6}$, Wael M. Refai ${ }^{7}$, Mohamed E. Ibrahim ${ }^{7}$, Mohamed G. Elshal ${ }^{7}$

${ }^{1}$ Department of Internal Medicine, Nephrology \& Renal Transplantation Division,

${ }^{2}$ Department of Medical Physiology, ${ }^{3}$ Department of Rheumatology \& Rehabilitation,

${ }^{4}$ Department of Medical Biochemistry \& Molecular Biology, ${ }^{5}$ Department of Diagnostic Radiology,

Faculty of Medicine, Assiut University, ${ }^{6}$ Department of Orthodontics, Faculty of Dentistry, Aswan University,

Egypt. ${ }^{7}$ Department of Orthodontics, Faculty of Dentistry, Minia University, Egypt

*Correspondence to Marwa Kamal Abdo Khairallah, Fax: 71515, Mobile: 002/01097878113, E-mail: marwa.kamal82@ hotmail.com

\section{ABSTRACT}

Purpose: Uremia influences bone remodeling. This study aimed to evaluate the impact of treatment with local injection of Vitamin $\mathrm{D}_{3}$ (Vit. $\mathrm{D}_{3}$ ) on bone remodeling associated with orthodontic treatment in uremic rabbits.

Patients and Methods: rabbits were split into four groups: a healthy control, a sham second group, where antibiotic was given in therapeutic dose, uremic third group, uremia induced by a nephrotoxic dose of antibiotic, and the fourth uremic group treated with locally injected Vit. $\mathrm{D}_{3}$. All groups experienced orthodontic procedures. Bone density before and after tooth movement was determined using quantitative computed tomography. Analysis of serum parathyroid hormone was done by Elisa while serum calcium, urea, and creatinine were done by enzymatic colorimetric assay. Maxillary bone underwent histological analyses. Results: A statistically significant reduction in bone density in uremic models $(\mathrm{P}<0.05)$ versus healthy models. Improvement in bone density in the treated uremic group after treatment with locally injected Vit. $\mathrm{D}_{3}$, which led to increase osteoblastic activity in comparison to the non-treated uremic group. A significant negative correlation was detected between serum calcium and bone density in the non-treated uremic group, this correlation was lost in the treated uremic group. Conclusions: Treatment of uremic models with locally injected Vit. $\mathrm{D}_{3}$, had significantly improved bone density by increasing osteoblastic and decreasing osteoclastic activity regardless of calcium and parathyroid levels. Local Vit. $\mathrm{D}_{3}$ could be used to improve bone remodeling in uremia.

Keywords: Uremia, bone remodeling, Dihydroxycholecalciferol, Osteoblastic chronic kidney disease.

\section{INTRODUCTION}

Recently orthodontic treatment is universally practiced in patients with uremia as a result of advancement in medical services resulting in reduced mortality in chronic kidney disease (CKD) patients. Clinical findings of CKD influence multiple organs. Bone disease is a universal feature of CKD (1). Calcium metabolism is compromised by an elevated parathyroid hormone and by the disruption in Vit.D metabolism that results in secondary hyperparathyroidism (2). Besides, persistent inflammatory processes are prevalent in CKD patients. This is associated with the presence of several factors, as uremic mediators, high levels of oxidative stress, protein-energy wasting, circulating proinflammatory cytokines, enhanced infections, and chronic inflammation evaluated by high levels of C-reactive protein ${ }^{(3)}$.Uremia acts as a bone metabolic disorder affecting bone manipulative proceedings such as orthodontic treatment. Still, experimental researches that examine the effect of uremia in CKD on orthodontics as representative of bone remodeling are scanty. Previous experimental studies on orthodontic treatment have proven that injection of $1,25(\mathrm{OH})_{2}$ dihydroxycholecalciferol (Vit.D ${ }_{3}$ ) and implementation of mechanical force stimulate formalization of several osteoclasts and increase bone resorption (4), bringing about higher rates of teeth movements than those produced only by mechanical force (5). Knowing that Vit. $\mathrm{D}_{3}$ affects bone metabolism in an anabolic manner, osteoblastic cells have high-affinity receptors for $V i t . D_{3}$, a finding that strengthens the idea of its regulatory bone formation and mineralization effect ${ }^{(6)}$. Also, it is well known that chronic kidney disease patients have Vit.D deficiency ${ }^{(7)}$. Accordingly, bone remodeling evaluated by the rate of bone formation concerning orthodontic tooth movement (OTM) in CKD patients with and without a combination of Vit. $\mathrm{D}_{3}$ seemed to be a point worthy investigation. So, this study is conducted to highlight this aim.

\section{MATERIALS AND METHODS}

Animals: The flow diagram (Fig. 1) is showing the steps in our study. The study included $45 \mathrm{New}$ Zealand healthy male rabbits, ranged from 10-12 months in age, and 2.5-3 $\mathrm{kg}$ in weight at the start of the experiment. They were housed in clean cages in the animal house, Faculty of Medicine, Assiut University. They were maintained on a natural 12:12-h light-dark cycle in an aerated room, temperature $\left(25 \pm 5^{\circ} \mathrm{C}\right)$, standard rabbit meals and water were available. Animals were habituated for handling and acclimatized to laboratory conditions for one week before the start of the experiment. At the start of the experiment they were randomly assigned into four groups: Healthy control group $(n=10)$, in which only OTM was conducted. Sham group: $(\mathrm{n}=11)$, in which only gentamycin injections were given in regular therapeutic doses $(3-5 \mathrm{mg} / \mathrm{kg} / \mathrm{day}$ IM). Non-treated uremic group (CKD group) $(n=12)$, in which nephropathy was induced by gentamycin, and treated uremic group (CKD Treated group) $(\mathrm{n}=12)$, which were treated by local injection of Vit. D3 after induction of nephropathy and then orthodontic treatment was done in all the four groups. 


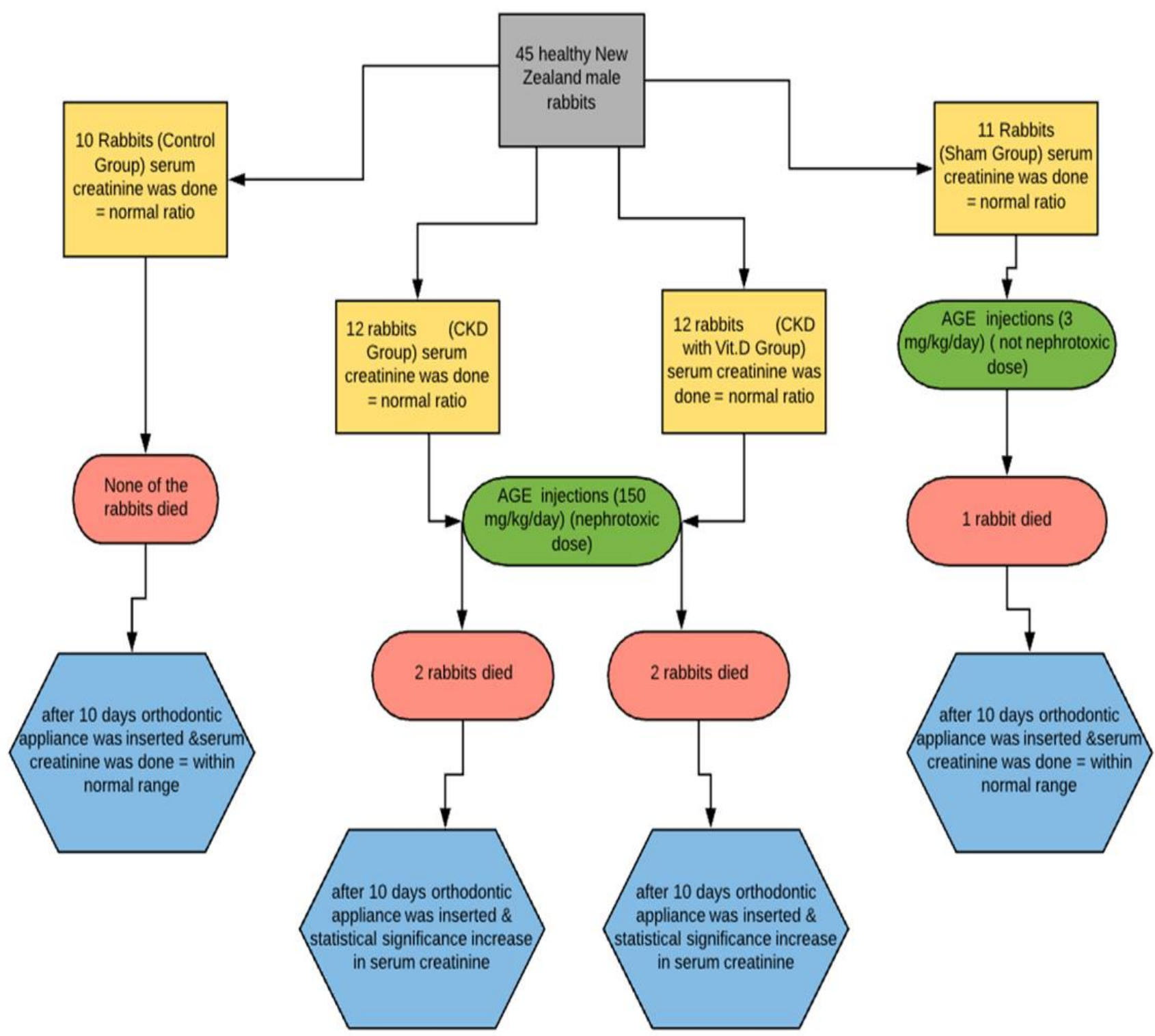

Fig (1): Flow diagram showing the main steps in our study

\section{Ethical consideration:}

- All experimental protocols and procedures were done following the standard outlined in guidelines for care and use of experimental animals by the Committee for Purpose of Control and Supervision of Experiment on Animals (CPCSEA), and according to the National Institute of Health (NIH) Protocol and were approved by Institutional Animal Care, Faculty of Medicine, Minia University: (number 290/2/7/2018).

- Laboratory methods were performed following relevant guidelines and regulations.

- This article does not contain any studies with human participants performed by any of the authors.

\section{Induction of nephropathy in rabbits:}

Aminoglycosides (AGE) (AGE-treated groups) were received at a dose of $150 \mathrm{mg} / \mathrm{kg} / \mathrm{day}$ by intraperitoneal injection. Nephropathy occurred after 10 days. After that, we kept injecting a maintenance dose of $20 \mathrm{mg} / \mathrm{kg} / \mathrm{day}$ to keep nephropathy state ${ }^{(\mathbf{8})}$.

\section{Application of orthodontic appliances:}

Twenty-one days after induction of uremia, all of the animal groups were anesthetized with a combination of ketamine hydrochloride $50 \mathrm{mg} / \mathrm{mL}$ (Ketamine) with xylazine hydrochloride $23.3 \mathrm{mg} / \mathrm{mL}$ (Xyla-ject) intramuscularly in the thigh muscle. An orthodontic appliance was placed in all groups. Appliances were made in concordance to that made by Abtahi et al. ${ }^{(9)}$. The appliance was formed of nickel-titanium closed-coil spring ligated and activated in between the mandibular right first premolar and central incisor thus delivering a force measured about $60 \mathrm{~g}$.

\section{Measurement of tooth movement and bone density:}

All measurements were obtained twice for all groups; one at the start of the study before insertion of the appliance, and one after removal of the appliance. Three weeks after appliance insertion, rabbits were anesthetized; the mesiodistal space between the distal surface of the mandibular right central incisor and the mesial surface of the mandibular right first premolar was measured radiographically using computerized tomography (CT).

Creatinine and parathyroid hormone (PTH) assays: 
Before the application of appliance and before sacrifice, blood samples were taken by the intra-cardiac approach. Serum calcium, urea, and creatinine levels were determined by enzymatic colorimetric kits. Catalog numbers for used kits are, CA12 10 bio-diagnostics for calcium, QuantiChrom ${ }^{\mathrm{TM}}$ Urea Assay Kit II (Catalog No: DUR2-100) for urea, and DetectX ${ }^{\mathrm{TM}}$ Serum Creatinine Kit Catalog No: KB02-H2 for determination of serum creatinine respectively. Serum PTH levels were measure by ELISA-PTH (CAT NO: MBS732169).

\section{Method and Dose of Vit.D3 administration:}

Twenty microliters of $10^{-10} \mathrm{~mol} / \mathrm{L} ; 1,25(\mathrm{OH})_{2} \mathrm{D}_{3}$; ACME Laboratories Ltd; were locally injected in submucosa mesial and distal to the apical root part of right premolar with a 30-gauge needle, once every 7 days. Injections were administered on days $0,7,14$, and day 21 .

\section{Fixed orthodontic appliance placement:}

The appliance was designed to move the right mandibular incisor distally against mandibular first premolars. The following steps were carried out: the mouth of the rabbit was opened using a Molt mouth gauge and cheek retractor to improve accessibility. Ligature wire of 0.014 inches was inserted distal to the maxillary first premolar and a Ni-Ti closed coil spring $(0.010 \mathrm{X}$ $0.003^{\prime \prime}$ and $12 \mathrm{~mm}$ length) with readymade stainless steel eyelets was inserted in one end of the ligature wire, then both ends of the ligature wire were approximated together and tightened using hemostat, then the excess wire was trimmed. Application of composite on tooth surface was made to prevent accidental wire dislodgement. The coil spring was stretched to apply a force of $60 \mathrm{gm} \pm 10$ on each side confirmed by the use of force gauge.

\section{Histological analyses}

Samples for histological studies were taken at the end of the experiment from the lower jaws of rabbits, fixed in neutral buffer formalin $10 \%$ for one week. Fixed samples were decalcified in formic acid after washing in phosphate buffer saline (PBS) to remove the used fixative. Decalcified samples for the paraffin section were trimmed and washed again to remove formic acid, dehydrated in ascending grades of ethanol, cleared in xylene, infiltrated and, embedded in paraffin wax. Serial sections of 5 microns were obtained and stained with hematoxylin and eosin $(\mathrm{H}$ and $\mathrm{E})$ as well as Masson trichrome stains according to the protocol described by Garcia-Donas $\boldsymbol{e t}$ al. ${ }^{(10)}$.

For electron microscopy, selected samples from the decalcified material were processed in the Electron Microscope Unit of Assiut University. Sections were placed on copper grids, contrasted with uranyl acetate and lead citrate, and analyzed in JEM 100 CX11 transemission microscopy (TEM). Photographs were taken by digital camera Model XR-41M with the same equipment connected to a DELL desktop computer ${ }^{(11)}$.

\section{Statistical analysis}

Recorded data were analyzed using the statistical package for social sciences, version 20.0 (SPSS Inc., Chicago, Illinois, USA). Quantitative data were expressed as mean \pm standard deviation (SD). Qualitative data were expressed as frequency and percentage. Independent-samples t-test of significance was used when comparing two means. Chisquare $\left(\mathrm{x}^{2}\right)$ test of significance was used to compare proportions between two qualitative parameters. The confidence interval was set to $95 \%$ and the margin of error accepted was set to $5 \%$. The p-value was considered significant as the following: P-value $<0.05$ was considered significant. P-value $<0.001$ was considered highly significant. P-value $>0.05$ was considered insignificant.

\section{RESULTS}

The average weight of included animals at the time of sacrifice was $2838.00 \pm 176.26 \mathrm{gm}$ in control, 2805.50 $\pm 151.12 \mathrm{gm}$ in sham, $2479.00 \pm 161.55 \mathrm{gm}$ in CKD, and $2452.00 \pm 151.06 \mathrm{gm}$ in CKD with locally injected Vit.D 3 with a significant statistical difference between CKD and healthy groups. For creatinine, the mean level at the time of sacrifice was $0.85 \pm 0.16$ in control, and $0.80 \pm 0.10$ in sham, $3.37 \pm 0.31$ in CKD group, $3.31 \pm 0.34$ in CKD with locally injected Vit. $\mathrm{D}_{3}$. The difference was significant between CKD and healthy groups $(\mathrm{P}<0.0001)$. Levels of PTH showed a non-statistical difference between CKD groups, however, there was a significant difference between CKD groups and healthy groups (Fig. 2). No statistical difference in distance between right central incisor and right first premolar in all four groups before orthodontic appliance. After appliance removal, there was a significant increase in OTM in CKD groups versus healthy groups. Furthermore, OTM was significantly increased in CKD versus CKD with Vit. $\mathrm{D}_{3}$ injection (Table 1).

Our results showed that the percentage of reduction in bone density measured by a Quantitative CT scan was lowest in control and sham group with no statistical difference between them, while the highest reduction in bone density was in CKD group in comparison to all groups (Table 2). Histological analyses showed that after OTM, the alveolar bone volume was significantly reduced in CKD group in comparison to CKD with Vit. $\mathrm{D}_{3}$ group $(\mathrm{P}<0.001)$ (Figs. 3-5), however, the osteoclastic surface was significantly increased in CKD group in comparison to other groups including CKD with Vit. $\mathrm{D}_{3}(\mathrm{P}<0.001)$, still osteoclastic surface increased significantly in both CKD groups versus healthy groups. Furthermore osteoblastic surface was significantly lower in CKD groups versus healthy groups, with a more significant decrease in the osteoblastic surface in CKD group versus $\mathrm{CKD}$ with Vit. $\mathrm{D}_{3}$ group (Figs 3-5). A significant negative correlation between serum calcium and CKD and the anticipated finding was the disappearance of this negative correlation in CKD with locally injected Vit. $\mathrm{D}_{3}$ (Table 3 ). 
Table (1): Orthodontic tooth movement (identified by measuring the distance between lower central incisor and the lower first premolar on the right side in $\mathbf{~ m m}$ ):

\begin{tabular}{|c|c|c|c|c|}
\hline & Control & Sham & CKD & CKD + Vit.D \\
\hline \multicolumn{5}{|l|}{ Pre: } \\
\hline Mean \pm SD & $27.35 \pm 1.08$ & $27.22 \pm 1.05$ & $27.37 \pm 1.15$ & $27.20 \pm 1.26$ \\
\hline Range(mm) & $25.6-29.6$ & $25.6-29.6$ & $25.3-29.6$ & $25.3-29.6$ \\
\hline $\mathrm{P}$-value ${ }^{1}$ & & 0.783 & 0.973 & 0.773 \\
\hline $\mathrm{P}$-value ${ }^{2}$ & & & 0.764 & 0.970 \\
\hline $\mathrm{P}$-value ${ }^{3}$ & & & & 0.756 \\
\hline \multicolumn{5}{|l|}{ Post: } \\
\hline Mean \pm SD & $26.69 \pm 1.13$ & $26.55 \pm 1.04$ & $22.83 \pm 0.95$ & $24.72 \pm 1.46$ \\
\hline Range(mm) & $24.8-29.1$ & $24.9-28.8$ & $21.6-24.8$ & $21.9-27.3$ \\
\hline P-value ${ }^{1}$ & & 0.783 & $<0.001 *$ & $0.003 *$ \\
\hline P-value ${ }^{2}$ & & & $<0.001 *$ & $0.005^{*}$ \\
\hline P-value ${ }^{3}$ & & & & $0.003 *$ \\
\hline P-value ${ }^{4}$ & $<0.001^{*}$ & $<0.001^{*}$ & $<0.001 *$ & $<0.001 *$ \\
\hline \multicolumn{5}{|l|}{ Difference: } \\
\hline Mean \pm SD & $0.67 \pm 0.11$ & $0.67 \pm 0.11$ & $4.54 \pm 0.78$ & $2.48 \pm 0.54$ \\
\hline Range $(\mathrm{mm})$ & $0.5-0.8$ & $0.5-0.8$ & $3.5-5.6$ & $1.8-3.4$ \\
\hline $\mathrm{P}$-value ${ }^{1}$ & & 0.951 & $<0.001 *$ & $<0.001^{*}$ \\
\hline P-value ${ }^{2}$ & & & $<0.001 *$ & $<0.001^{*}$ \\
\hline P-value ${ }^{3}$ & & & & $<0.001 *$ \\
\hline Percentage of OTM & $2.4 \%$ & $2.5 \%$ & $16.6 \%$ & $9.1 \%$ \\
\hline
\end{tabular}

P-value1: between control group and (sham, CKD, or CKD with Vit. $\mathrm{D}_{3}$ ) Groups

P-value2: between sham group and both the CKD groups.

P-value 3: between the CKD group and the CKD with local Vit.D 3 injected group.

P-value4: comparing orthodontic movement pre and post orthodontic appliance in each group separately (by measuring the distance between the lower central incisor and the lower first premolar on the right side).

Table (2): Bone density in all groups:

\begin{tabular}{|c|c|c|c|c|}
\hline & Control & Sham & CKD & CKD + Vit.D \\
\hline \multicolumn{5}{|l|}{ Pre: } \\
\hline Mean \pm SD & $447.69 \pm 50.59$ & $431.15 \pm 30.91$ & $433.39 \pm 22.22$ & $433.05 \pm 38.73$ \\
\hline Range & $370.6-520.7$ & $385.6-480.5$ & $403.8-470.4$ & $373.8-490.5$ \\
\hline $\mathrm{P}$-value ${ }^{1}$ & & 0.389 & 0.424 & 0.477 \\
\hline P-value ${ }^{2}$ & & & 0.854 & 0.905 \\
\hline P-value ${ }^{3}$ & & & & 0.981 \\
\hline \multicolumn{5}{|l|}{ Post: } \\
\hline Mean \pm SD & $332.05 \pm 50.91$ & $315.15 \pm 29.57$ & $117.72 \pm 21.43$ & $277.64 \pm 59.15$ \\
\hline Range & $257.2-410.1$ & $274.4-365.1$ & $85.0-149.8$ & $166.7-349.9$ \\
\hline P-value ${ }^{1}$ & & 0.376 & $<0.001 *$ & $0.041^{*}$ \\
\hline P-value ${ }^{2}$ & & & $<0.001 *$ & 0.090 \\
\hline P-value ${ }^{3}$ & & & & $<0.001 *$ \\
\hline P-value ${ }^{4}$ & $<0.001 *$ & $<0.001^{*}$ & $<0.001$ * & $<0.001^{*}$ \\
\hline \multicolumn{5}{|l|}{ Difference: } \\
\hline Mean \pm SD & $115.64 \pm 3.77$ & $116.00 \pm 3.01$ & $315.67 \pm 30.59$ & $155.41 \pm 53.29$ \\
\hline Range & $110.6-120.8$ & $111.2-120.2$ & $274.6-380.7$ & 90.4-260.7 \\
\hline P-value ${ }^{1}$ & & 0.816 & $<0.001 *$ & $0.030^{*}$ \\
\hline $\mathrm{P}$-value ${ }^{2}$ & & & $<0.001 *$ & $0.031 *$ \\
\hline $\mathrm{P}$-value ${ }^{3}$ & & & & $<0.001 *$ \\
\hline $\begin{array}{l}\text { Percentage of reduction } \\
\text { in bone density }\end{array}$ & $25.8 \%$ & $26.9 \%$ & $72.8 \%$ & $35.8 \%$ \\
\hline
\end{tabular}

P-value1: between control group and (sham, CKD, or CKD with Vit.D 3 ) Groups

P-value2: between sham group and both the CKD groups.

P-value 3: between the CKD group and the CKD with local Vit. $\mathrm{D}_{3}$ injected group.

P-value 4: comparing bone density pre and post orthodontic appliance in each group separately (by measuring the mesiodistal space between the distal surface of the mandibular right central incisor and the mesial surface of the mandibular right first premolar was measured radio-graphically using computerized tomography). 


\section{https://ejhm.journals.ekb.eg/}

Table (3): Correlation of orthodontic tooth movement and bone density with serum creatinine (mg/dl), PTH $(\mathrm{pg} / \mathrm{ml})$ and serum calcium $(\mathrm{mg} / \mathrm{dl})$ at the time of sacrifice in all groups:

\begin{tabular}{|c|c|c|c|c|c|}
\hline \multirow{2}{*}{ Groups } & & \multicolumn{2}{|c|}{ Drthodontic tooth movement } & \multicolumn{2}{|c|}{ Bone density } \\
\hline & & r-value & P-value & r-value & P-value \\
\hline \multirow{3}{*}{ Control $(\mathrm{N}=10)$} & Serum creatinine & -0.374 & 0.287 & 0.146 & 0.688 \\
\hline & PTH & -0.343 & 0.332 & 0.083 & 0.821 \\
\hline & Serum calcium & -0.370 & 0.292 & 0.183 & 0.612 \\
\hline \multirow{3}{*}{ Sham $(N=11)$} & Serum creatinine & -0.156 & 0.667 & -0.027 & 0.941 \\
\hline & PTH & -0.207 & 0.566 & 0.015 & 0.967 \\
\hline & Serum calcium & -0.138 & 0.704 & 0.078 & 0.830 \\
\hline \multirow{3}{*}{ CKD $(\mathrm{N}=12)$} & Serum creatinine & 0.656 & 0.039* & -0.714 & 0.020* \\
\hline & PTH & -0.383 & 0.275 & 0.124 & 0.733 \\
\hline & Serum calcium & -0.894 & $<0.001 *$ & 0.744 & 0.014* \\
\hline \multirow{3}{*}{$\begin{array}{l}\text { CKD + Vit.D } \\
(\mathrm{N}=12)\end{array}$} & Serum creatinine & 0.916 & $<0.001 *$ & -0.714 & $0.020 *$ \\
\hline & PTH & -0.197 & 0.585 & 0.423 & 0.223 \\
\hline & Serum calcium & -0.675 & 0.032* & 0.531 & 0.114 \\
\hline
\end{tabular}
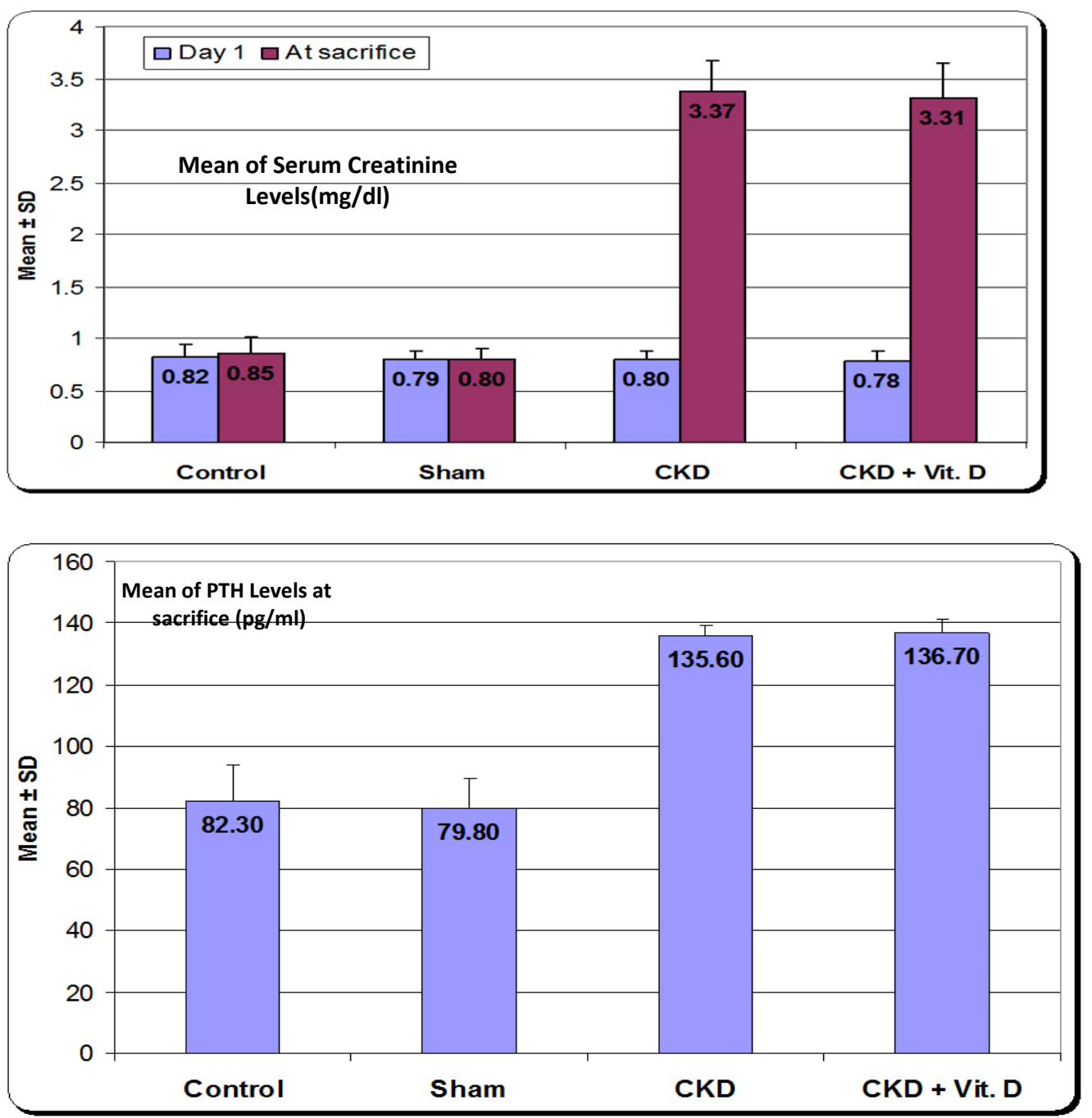
https://ejhm.journals.ekb.eg/

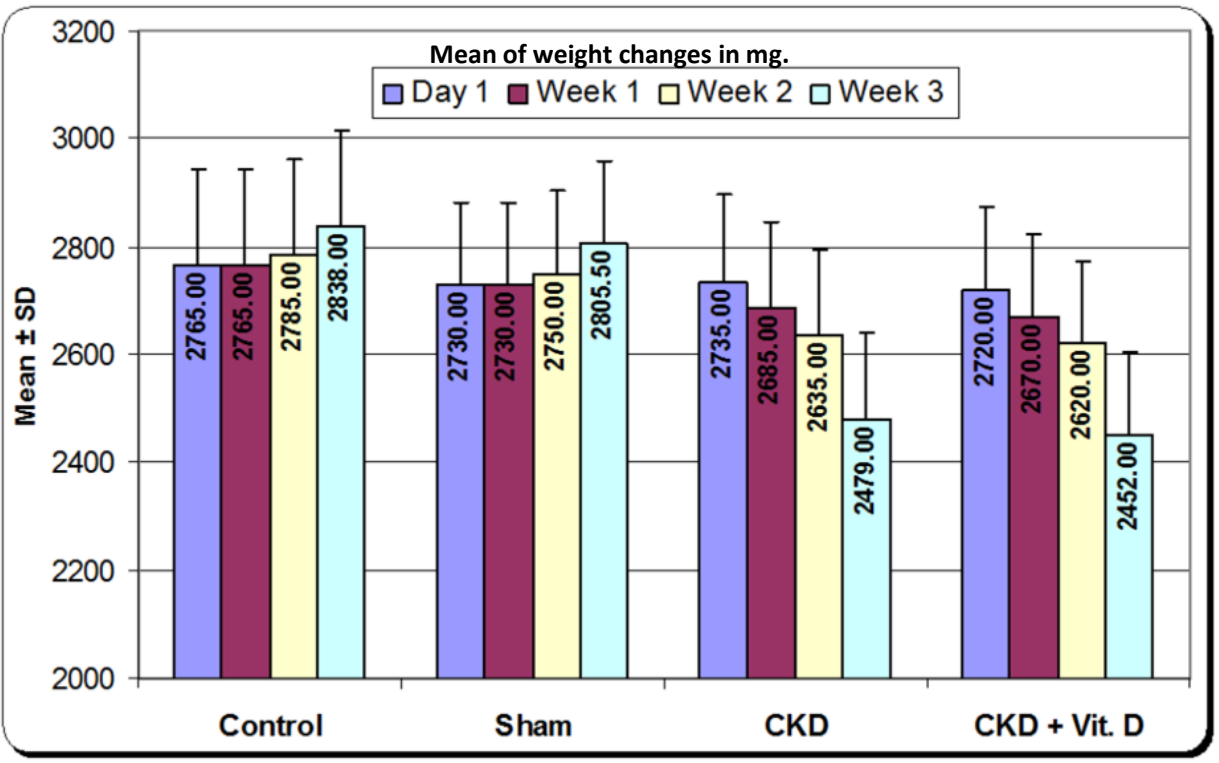

Fig (2): Clustered column chart for the mean values of serum creatinine level (mg/dl), PTH (Pg/ml), and weight changes $(\mathrm{mg})$ in different groups.

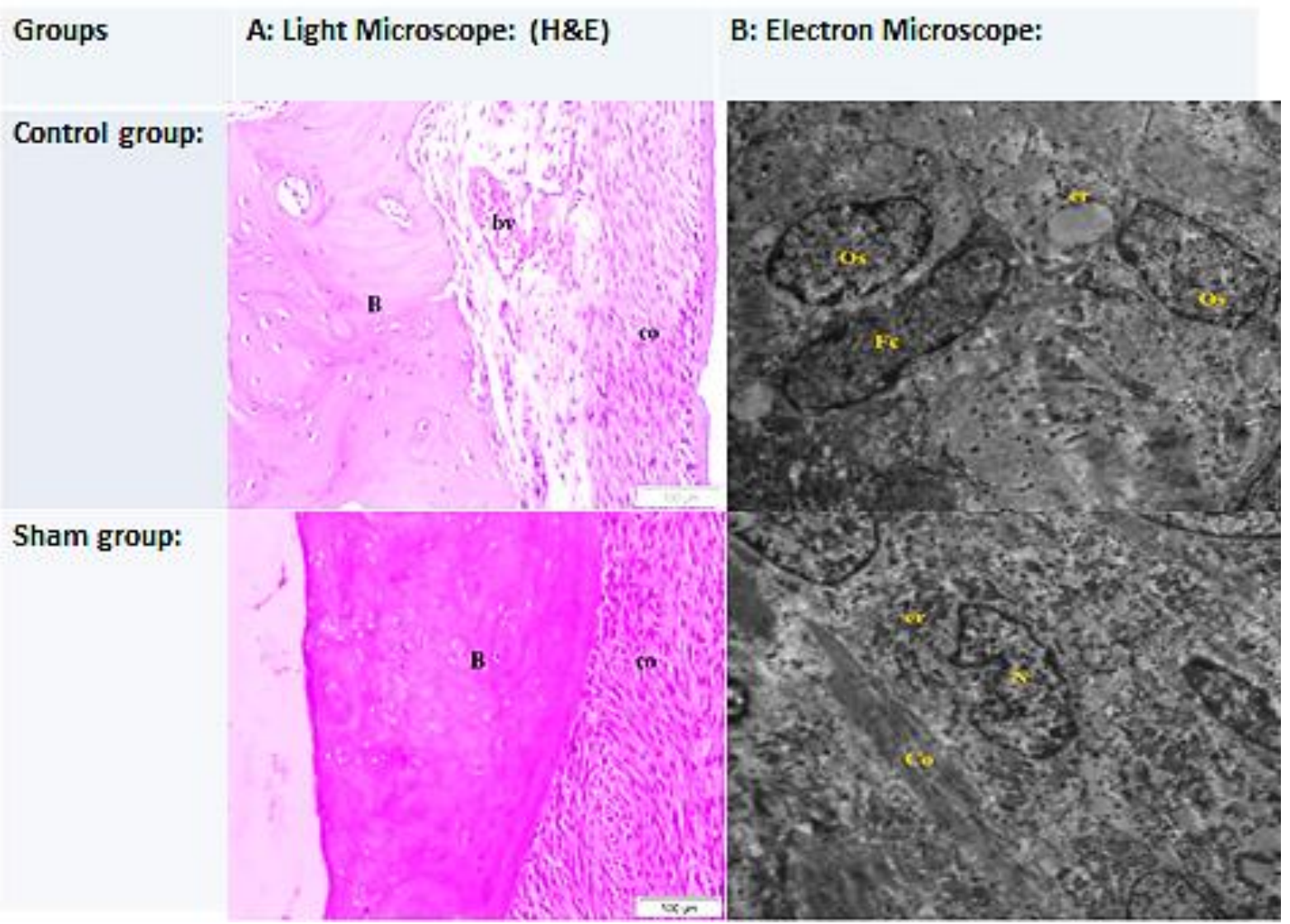

Fig (3): Histological analyses of bone specimens in control and sham groups by both light and electron microscope:

Bone specimens obtained from right lower mandibular bone including bone surrounding central incisor and first premolar. Magnification power of Light microscope images: (H\&E: X100), scale bar: 100um.

Magnification power of Electron microscope images: 2 microns, HV=80.0kv, Direct Mag: 3600X, Print Mag: 3600x86mm.

The control group: Light microscope by $\mathrm{H}$ and $\mathrm{E}$ stain from pressure side is showing periodontal ligament formed by mature collagenous tissue (co) with congested blood vessels (bv) and well-developed compact bone.

The electron microscopy of the periosteum is showing differentiation of fibroblasts $(\mathrm{Fc})$ to osteoblasts $(\mathrm{Os})$ having dilated rough endoplasmic reticulum (RER) (er) containing light electron-dense material. Besides, bony parts are showing osteoblasts (Os) rich with free ribosomes (r) and arranged in a lamellar manner

Sham group: $\mathrm{H}$ and $\mathrm{E}$ stain by light microscope for the paraffin section from tension side is showing bony part (B) thickening and collagenous fibers (co) that form periodontal ligaments.

The electron microscopy of the periosteum of the tension side is showing proliferating osteoblasts (Os) embedded in a ground matrix (X) and collagen fiber (co). Besides bony parts of the pressure side is showing osteocytes having dentate nucleus $(\mathrm{N})$ and rich with RER (er) and ground substance contain collagen fiber (co). 


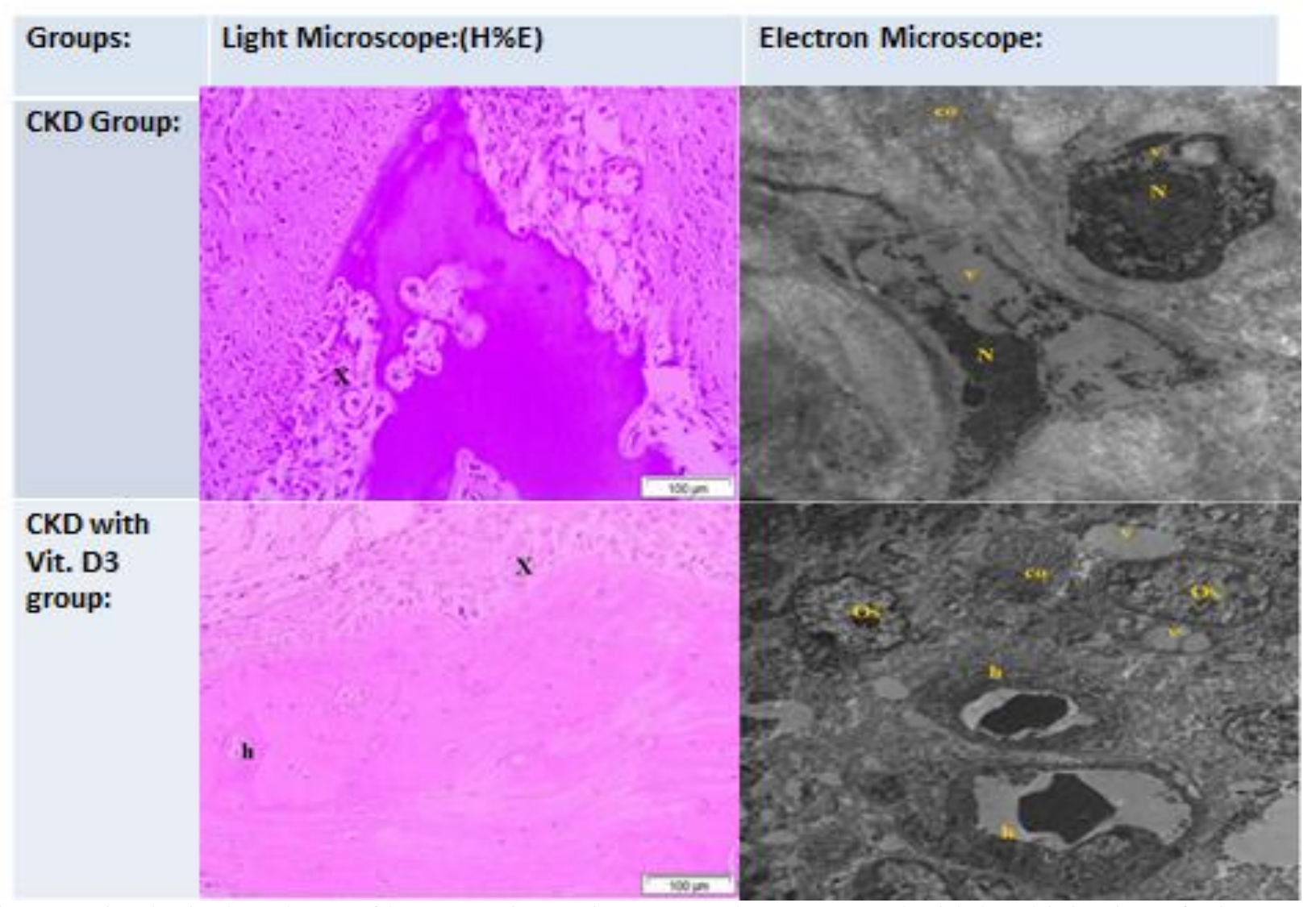

Fig (4): Histological analyses of bone specimens in CKD and CKD treated with local injection of VitD3 groups by both light and electron microscope:

Bone specimens obtained from the right lower mandibular bone including bone surrounding the central incisor and first premolar.

The magnification power of Light microscope images: (H\&E: X100), scale bar: 100um.

Magnification power of Electron microscope images: 2 microns, HV=80.0kv, Direct Mag: 3600X, Print Mag: 3600x86mm.

CKD group: Light micrograph of paraffin section by $\mathrm{H}$ and $\mathrm{E}$ stain from bony parts of tension side is showing the presence of osteolytic areas (x) with near absence of the Haversian system.

Electron micrograph of bony parts of both sides is showing cytoplasm of differentiated fibroblast to osteoblasts contains variable size vacuoles (v) in their cytoplasm and pyknosis of their nucleus $(\mathrm{N})$. The ground matrix is formed by light electron-dense material with the presence of collagen fibers (co). Besides, bony parts of the pressure side are showing numerous osteoblasts aggregated to each other having missed shaped nucleus (N) and their cytoplasm having numerous small vacuoles (v) and RER (er). Notice the ground substance formed by light electron-dense matrix $(\mathrm{X})$.

CKD group injected with local submucosal Vit. $\mathrm{D}_{3}$ : Light micrograph by $\mathrm{H}$ and $\mathrm{E}$ stain of paraffin section from tension side is showing collagen fiber of periodontal ligaments edematous and faintly stained (x). Notice the bony parts contain few Haversian canaliculi (h).

Electron micrograph from bony parts of the tension side is showing osteoblasts contain variable size vacuoles contain light electron material (v), RER (er), and dentate nucleus $(\mathrm{N})$, and ground substance contain collagen (co). Besides, bony parts of both sides are showing osteoblast (Os) containing dentate elongated nucleus $(\mathrm{N})$ and their cytoplasm contain variable size dilated vacuoles (v) contain light electron-dense material, The ground substance contains variable size electron-dense lysosomes, it also shows osteoblast cells (Os) forming Haversian system tend to arrange around canaliculi (h) and containing variable size vacuoles (v) with the presence of collagen fiber in matrix (co). 


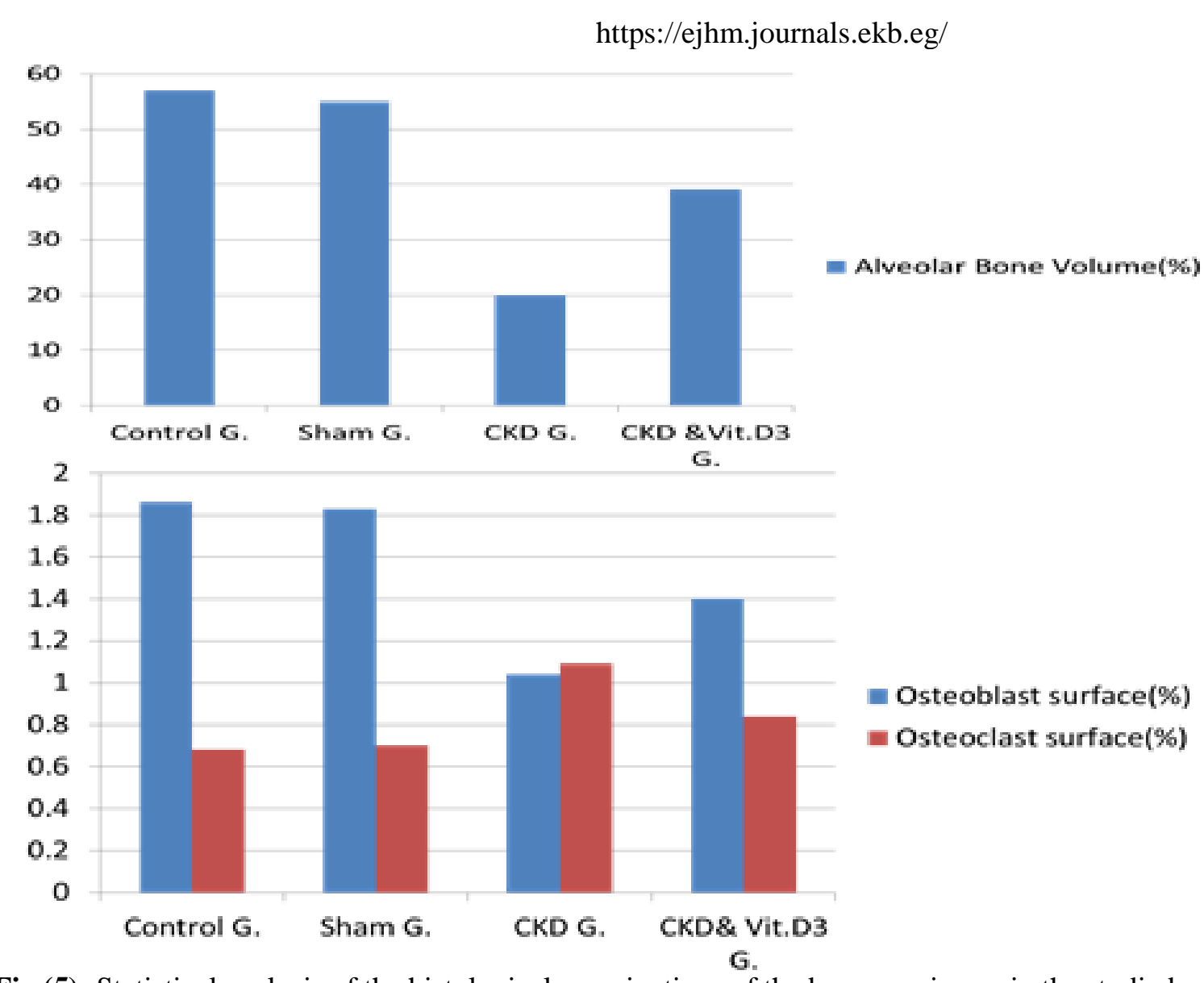

Fig (5): Statistical analysis of the histological examinations of the bone specimens in the studied animal groups including 5A: analysis of the alveolar bone volume,5B: analysis of the Osteoblastic and Osteoclastic surfaces.

After orthodontic appliance, the alveolar bone volume in both CKD groups was less than in the control and sham groups $(\mathrm{P}<0.001)$. Also, a significant decrease in alveolar bone volume was observed in the CKD versus the CKD with the local Vit.D3 injected group. Besides, the osteoblast surfaces were significantly lower in the CKD groups versus the control and sham groups $(\mathrm{P}<0.001)$. Still, the osteoblastic surface is significantly lower in the CKD group in comparison to the CKD with Vit.D3 injected group. Finally, the osteoclast surfaces increased in the CKD groups compared with the control and sham groups $(\mathrm{P}<0.001)$. The $\mathrm{CKD}$ group showed the highest significant osteoclastic surface among all groups even compared with the CKD with locally injected Vit.3 D group $(\mathrm{P}<0.001)$. However, there were no significant differences between the control group and the sham group in all analyses.

\section{DISCUSSION}

In this current study, the validity of the chronic kidney disease model used in this study was proven by serum biochemical changes. Within 10 to 15 days after renal nephrotoxicity, CKD groups exhibited elevated serum creatinine. Other biochemical markers of uremic condition were also in agreement with former studies published by other authors using similar CKD rabbit models (12). Subsequently, this animal model was compatible to simulate metabolic disturbances in CKD patients. Boudet et al. ${ }^{(13)}$, claimed that there is no need to start renal supportive intervention as this method of induction of renal disease produces long-lasting changes of renal function evolution pattern, that take several months to evoke chronic renal failure that needs special diet and supportive care.

Orthodontic tooth movement depends on arranged tissue resorption and figuration in the surrounding bone and periodontal ligament. An aseptic inflammatory cascade starts as a result of regional hypoxia associated with tooth load resulting in osteoclast resorption in sites of compression and osteoblast precipitation in sites of tension. Therefore OTM could be used as a model for assessment of bone remodeling process ${ }^{(\mathbf{1 2})}$. It is well known that uremia is associated with compromised bone health. To the best of our knowledge, the current study evaluates for the first time impact of progressive uremia with and without treatment with local injection of Vit.D3 on bone remodeling associated with OTM in vivo. We found that impaired renal function led to decrease bone regenerative capacity and increase degenerative capacity, suggesting that increased bone degenerative capacity was formed when uremia was existed, while treatment with local injection of Vit.D3 in those uremic models led to the improvement in bone regenerative capacity ${ }^{\mathbf{1 4})}$.

We used quantitative-CT analyses which showed a significant reduction in bone thickness in CKD groups in comparison to non-uremic groups, with dramatic bone density reduction occurring in CKD without Vit. $\mathrm{D}_{3}$ group. Our study results regarding the effectiveness of quantitative $\mathrm{CT}$ in measuring bone density before and after OTM was in agreement with other researchers who showed that measurement of bone mass density in CKD patients by quantitative CT is considered to be a useful tool because CT scan could differentiate between cortical and trabecular bone and more recently CT scan allows 
visualization of ultrastructural details that improve its ability to predict fractures in CKD patients (15). Furthermore, it estimates the quantity and intensity of inorganic matrix inside bones, and so it is considered as a perfect evaluator of fracture risk ${ }^{(16)}$. In contrary to our findings Russell and Avioli ${ }^{(17)}$ documented progressive disturbances in bone collagen within 15 days next to the inducement of CKD. However, the levels of bone minerals remain the same. This controversy could be simply attributed to the efficacy of CT scan which is more accurate than the radiographic films used by previously mentioned authors in measuring bone density in CKD patients ${ }^{(\mathbf{1 5})}$, although, in our study results, changes in bone density measured by CT correlated with electrolyte abnormalities associated with uremia.

Worth noting, that if CKD persisted in those models used by Russell and Avioli ${ }^{(17)}$ there would be more loss in amount and density of bone matrix sufficient to appear on radiographic films. Our results differed also from those of Shirazi et al. ${ }^{(18)}$ who found that bone density did not differ statistically in CKD group versus control and sham groups despite the statistically significant difference in the OTM. This controversy could be explained by the different ways of induction of uremia where medical nephrotoxicity used in our study offers more time for CKD bone-related bio-alterations to happen. Another explanation for this controversy is that bone volume in the latter study was evaluated by optical densitometry skull radiographs. This method is known to have small measuring range and also it is operator dependent, therefore it is of limited accuracy ${ }^{(\mathbf{1 9})}$.

Our results regarding histological evaluation showed increased osteoclast surface and significant decreased alveolar bone volume in uremic groups (Figs. 3-5). These histological findings were more dramatic in the CKD group without Vit. $\mathrm{D}_{3}$ injection. These data indicate that uremia, especially in the absence of local Vit. $D_{3}$, has dramatic effects on the bones of the oral cavity. In agreement with our results, Pan and Loke ${ }^{(20)}$ found a significant decrease in bone density in uremic patients. In our study, this decrease was associated with an increased rate of OTM in both CKD groups in comparison to healthy groups.

Our results showed that decreased alveolar bone density occurred after activation of orthodontic appliance had led to tooth movement, however, impairment of biological bone processes was enough in CKD groups to produce a statistically significant higher rate of tooth movement compared to healthy groups. This significant increase in tooth movement in the CKD group without treatment could be explained by the significant decrease in bone density that appeared in both CT scan and histological analyses.

Our study showed no statistical difference between control and sham groups in the amount of tooth movement, which indicates that aminoglycosides in therapeutic dose have no bone effects and also it confirms that CKD groups differed from them because of uremia especially with the presence of equal appliance force in all models. Interestingly, the difference in alveolar bone volume between CKD groups and healthy groups during OTM persisted, even when treatment with a local injection of Vit. $D_{3}$ occurred. The decrease in alveolar bone density was more pronounced than in healthy groups, this could be attributed to the increase of inflammatory mediators in uremia, which causes more osteoclast differentiation ${ }^{(21)}$.

Other studies stated that other uremic toxins suppress bone formation and stimulate bone resorption besides hypocalcemia, hyperparathyroidism, abnormal activation of Vit. $\mathrm{D}_{3}$ end products, and chronic academia (22). Another important finding in our study was a negative correlation between serum calcium in our in vivo CKD models and increased rate of OTM which emphasize that hypocalcemia plays important role in increasing degenerative capacity during bone remodeling in uremic models. These results coincide with the results of previous researches where a higher rate of orthodontic movements was found in dogs having high phosphorus and low calcium-based nutrition, as well as calcium-free fed lactating rats ${ }^{(17)}$. Interestingly our study showed that when we treated CKD group with a local injection of Vit. $D_{3}$ the positive correlation between serum calcium level and bone density was lost, still, the negative correlation between calcium level and rate of OTM was preserved which emphasizes the presence of other players beside calcium and Vit.D3 levels in the pathogenesis of bone remodeling in uremic models ${ }^{\mathbf{2 2} 2)}$.

The anticipated finding regarding the effect of local Vit. $\mathrm{D}_{3}$ in increasing bone regeneration during orthodontic treatment in CKD patients is in concordance with the results of Hong $\boldsymbol{e t}$ al. ${ }^{(23)}$ who examined the effects of Vit.D3 on bone regeneration in dogs. Other researchers as Almoammar found that Vit. $\mathrm{D}_{3}$ injection increased bone formation and thus positively impacted bone remodeling by increasing osteoblastic activity ${ }^{(24)}$. In contrary to our results, other researchers found that local injections of Vit.D caused an increase in the number of osteoclasts, and therefore increased bone resorption, increasing the rate of tooth movement ${ }^{(25)}$. The discrepancy in these results could be attributed to different doses of local Vit. $\mathrm{D}_{3}$ used in each research and also the difference in the levels of native Vit.D used and whether there was Vit.D deficiency in these models or not.

From the previous results, we postulated that local Vit. $\mathrm{D}_{3}$ could be considered to be safer and appropriate for improving bone remodeling in uremic patients than using antiresorptive treatment especially with the progressive decline in the kidney functions ${ }^{(26)}$.

\section{CONCLUSION}

Currently, data about bone remodeling with uremia are limited. Because of increased demand from adult CKD patients, for bone remodeling required treatment as orthodontics, it is necessary to understand the bone alterations in those patients. Our study showed that diminished bone formation and decreased bone density were observed in uremia suggesting that bone remodeling 
is altered. Our results concluded that these alterations could be minimized by local injection of Vit. $\mathrm{D}_{3}$.

\section{DECLARATIONS}

Conflict of interests: The authors declare that they have no conflict of interest.

Funding support: No funding resources were available. This research was completely funded by authors and did not receive any specific grant from any funding agencies

Consent for publication: not applicable -Availability of data and material: All related data and materials are available on request.

\section{REFERENCES}

1. Costantinides F, Castronovo G, Vettori E et al. (2018): Dental Care for Patients with end-stage renal disease and undergoing hemodialysis. https://doi.org/10.1155/2018/9610892.

2. Isakova T, Cai X, Lee J et al. (2020): Longitudinal Evolution of Markers of Mineral Metabolism in Patients With CKD: The Chronic Renal Insufficiency Cohort (CRIC) Study. American Journal of Kidney Diseases, 75(2): 235244.

3. Mihai S, Codrici E, Popescu I et al. (2018): Inflammation and chronic kidney disease: current approaches and recent advances. https://doi.org/10.1155/2018/2180373

4. Anand N, Chandrasekaran S, Alam M (2013): The malnutrition inflammation complex syndrome-the micsing factor in the perio-chronic kidney disease interlink. Journal of clinical and Diagnostic Research: JCDR., 7(4): 763-66.

5. Bartzela $\mathbf{T}$, Maltha $\mathbf{J}$ (2016): Medication effects on the rate of orthodontic tooth movement. In Biology of Orthodontic Tooth Movement, Springer, Cham, Pp. 133-159.

6. De Paula F, Rosen C(2010): Back to the future: revisiting parathyroid hormone and calcitonin control of bone remodeling. Hormone and Metabolic Research, 42(05): 299306.

7. Jean G, Souberbielle J, Chazot C (2017): Vitamin D in chronic kidney disease and dialysis patients. Nutrients, 9(4): 328-32.

8. Randjelovic P, Veljkovic S, Stojiljkovic N et al. (2017): Gentamicin nephrotoxicity in animals: Current knowledge and future perspectives. EXCLI Journal, 16: 388-93.

9. Abtahi M, Saghravanian N, Poosti M et al. (2018): Histological evaluation of orthodontic tooth movement following low level laser irradiation in rabbits. Electronic Physician, 10(1): 6219-23.

10.Garcia-Donas J, Dalton A, Chaplin I et al. (2017): A revised method for the preparation of dry bone samples used in histological examination: Five simple steps. Homo, 68(4): 283-288.

11.Shah F, Ruscsák K, Palmquist A (2019): 50 years of scanning electron microscopy of bone-a comprehensive overview of the important discoveries made and insights gained into bone material properties in health, disease, and taphonomy. Bone Research, 7(1): 1-15.

12.Li Y, Jacox L, Little S et al. (2018): Orthodontic tooth movement: The biology and clinical implications. The Kaohsiung Journal of Medical Sciences, 34(4): 207-214.

13. Boudet J, Man N, Pils P et al. (1978): Experimental chronic renal failure in the rat by electrocoagulation of the renal cortex. Kidney International, 14(1): 82-86.

14. Rehman K, Akash M, Azhar S et al. (2012): A biochemical and histopathologic study showing protection and treatment of gentamicin-induced nephrotoxicity in rabbits using vitamin C. African Journal of Traditional, Complementary and Alternative Medicines, 9(3): 360-365.

15.Shewale P, Aglawe V, Patta R et al. (2017): Techniques used for bone density measurement. Int J Comput Appl., 178: 2023.

16.Bover J, Ureña-Torres $P$, Torregrosa $J$ et al. (2018): Osteoporosis, bone mineral density and CKD-MBD complex (I): Diagnostic considerations. Nefrología (English Edition), 38(5): 476-490.

17.Russell J, Avioli L (1972): Effect of experimental chronic renal insufficiency on bone mineral and collagen maturation. The Journal of Clinical Investigation, 51(12): 3072-3079.

18.Shirazi M, Khosrowshahi M, Dehpour A (2001): The effect of chronic renal insufficiency on orthodontic tooth movement in rats. The Angle Orthodontist, 71(6): 494-498.

19. Iseri K, Dai L, Chen $Z$ et al. (2020): Bone mineral density and mortality in end-stage renal disease patients. Clinical Kidney Journal, 13(3): 307-321.

20.Pan B, Loke S (2018): Chronic kidney disease associated with decreased bone mineral density, uric acid and metabolic syndrome. Plos One, 13(1): 190-195.

21. Cafiero C, Gigante M, Brunetti G et al. (2018): Inflammation induces osteoclast differentiation from peripheral mononuclear cells in chronic kidney disease patients: crosstalk between the immune and bone systems. Nephrology Dialysis Transplantation, 33(1): 65-75.

22. Watanabe K, Tominari T, Hirata M et al. (2017): Indoxyl sulfate, a uremic toxin in chronic kidney disease, suppresses both bone formation and bone resorption. FEBS Open Bio., 7(8): 1178-1185.

23.Hong H, Yen T, Hong A, Chou T (2015): Association of vitamin D3 with alveolar bone regeneration in dogs. Journal of Cellular and Molecular Medicine, 19(6): 1208-1217.

24.Almoammar K (2018): Vitamin D and orthodontics: an insight review. Clinical, Cosmetic and Investigational Dentistry, 10: 165.

25.Pradeep R, Kumar S, Kannan M (2019): Role of VitaminD in Orthodontics: A Review. Indian Journal of Public Health Research \& Development, 10(12): 1085-89.

26.Bover J, Ureña-Torres $P$, Alonso A et al. (2019): Osteoporosis, bone mineral density and CKD-MBD (II): Therapeutic implications. Nefrología (English Edition), 39(3): 227-242. 